\title{
A CONVERGENCE THEOREM FOR LIMITÄRPERIODISCH T-FRACTIONS OF RATIONAL FUNCTIONS
}

\section{KARI HAG}

ABstract. We prove that a limitärperiodisch $T$-fraction, which corresponds to a rational function, converges (locally uniformly) to the original function in a certain domain.

1. Introduction. The sequence $\left\{A_{n}(z) / B_{n}(z)\right\}$ obtained by the rule

$$
\frac{A_{n}(z)}{B_{n}(z)}=1+d_{0} z+\frac{z}{1+d_{1} z}+\cdots+\frac{z}{1+d_{n} z}
$$

is called a $T$-fraction (see [5]). Moreover, the $T$-fraction is called limitärperiodisch if the sequence $\left\{d_{n}\right\}$ converges.

The $T$-fraction is said to converge for a certain $z$-value, if for that particular value

$$
\lim _{n \rightarrow \infty}\left(1+d_{0} z+\frac{z}{1+d_{1} z}+\cdots+\frac{z}{1+d_{n} z}\right)
$$

exists in $C$.

The $T$-fraction is said to correspond to the power series $(*) 1+\sum_{n=1}^{\infty} c_{n} z^{n}$ if (*) agrees with the power series expansion of $A_{n}(z) / B_{n}(z)$ up to and including the term $c_{k(n)} z^{k(n)}$, where $k(n) \rightarrow \infty$ as $n \rightarrow \infty$.

For every formal power series $1+\sum_{n=1}^{\infty} c_{n} z^{n}$, and thus for every function $f_{0}$, holomorphic in some region containing the origin, and normalized by $f_{0}(0)=1$, there is exactly one corresponding $T$-fraction. (A proof is given in [5].)

Starting with the function $f_{0}$, we obtain the $T$-fraction expansion in the following way:

Let $\left\{f_{n}\right\}$ be the sequence of functions, defined by

$$
\begin{aligned}
f_{n}(z) & =1+\left(f_{n}^{\prime}(0)-1\right) z+\frac{z}{f_{n+1}(z)}, \quad z \neq 0, n=0,1,2, \cdots, \\
f_{n+1}(0) & =1 .
\end{aligned}
$$

Received by the editors October 2, 1970 and, in revised form, May 20, 1971. AMS 1970 subject classifications. Primary 30A22.

Key words and phrases. Continued fractions corresponding to power series, convergence of continued fractions, $T$-fraction expansion, limitärperiodisch $T$-fraction.

(c) American Mathematical Society 1972 
With

$$
d_{n}=f_{n}^{\prime}(0)-1, \quad n=0,1,2, \cdots,
$$

the continued fraction (1) is the $T$-fraction of $f_{0}$.

Due to the linearity of the elements of the $T$-fraction a great deal can be said about the convergence. Several convergence theorems are proved in [2], [3], and [5]. The criteria are given in terms of conditions on the sequence $\left\{d_{n}\right\}$. A different kind of result is proved in [6], where convergence properties of the $T$-fraction expansion is concluded from boundedness conditions of the function. A step in the proof is to establish the following lemma (see [6, p. 8]):

LEMMA 1. Let $f_{0}$ be holomorphic in $|z|<1$, normalized by $f_{0}(0)=1$, and such that the function $f_{n}$, defined in (2) all are holomorphic in $|z|<1$. Further, let $f_{0}$ have a $T$-fraction expansion where $d_{n} \rightarrow-1$ as $n \rightarrow \infty$. Then the $T$-fraction of $f_{0}$ converges to $f_{0}$ uniformly on any compact subset of the open unit disk.

For rational $f_{0}$ this result can be extended in the following way (announced in [1]):

\section{The main result.}

THEOREM 1. Let $f_{0}$ be a rational function normalized by $f_{0}(0)=1$ and with limitärperiodisch $T$-fraction. Take an arbitrary $\theta \in(0,1)$ and let $D_{\theta}$ denote the disk $\{z ;|z| \leqq \theta\}$. Remove from $D_{\theta}$ arbitrary neighborhoods of the poles of $f_{0}$ in $D_{\theta}$. Then the $T$-fraction of $f_{0}$ converges to $f_{0}$ uniformly on the remaining set $D_{\theta}^{*}$.

REMARK 1. In this theorem the interval $(0,1)$ cannot be replaced by $(0, r)$ where $r \geqq 1$, as may be seen from the classic example $f_{0}=1$. (The $T$-fraction of this function has the form

$$
1-z+\frac{z}{1-z}+\cdots+\frac{z}{1-z}+\cdots
$$

and converges in $|z|<1$ to 1 , in $|z|>1$ to $-z$ and diverges on the unit circle, except for $z=-1$, where it converges to 1).

REMARK 2. The existence of an uncountable set of rational functions with nontrivial limitärperiodisch $T$-fractions is proved in [1]. Applying the functions used in this proof we can prove the existence of (an uncountable set of) rational functions with poles in $|z|<1$ and with nontrivial limitärperiodisch $T$-fractions.

For such functions the $T$-fraction expansion converges in a larger domain than the power series expansion. To prove Theorem 1 we state some 
3. Preliminary results. From now on we consider a normalized rational function $f_{0}$, i.e. let $f_{0}$ in $\S 1$ be given by the formula

$$
f_{0}(z)=\frac{1+\sum_{k=1}^{m_{0}} \beta_{k}^{(-1)} z^{k}}{1+\sum_{k=1}^{m_{0}} \beta_{k}^{(0)} z^{k}},
$$

where $\beta_{k}^{(-1)}, \beta_{k}^{(0)}$ are arbitrary (complex) constants, and let $\left\{f_{n}\right\}$ and $\left\{d_{n}\right\}$ be the sequences defined in (2) and (3) respectively. Then, for $n=1,2$, $3, \cdots$, we have

$$
f_{n}(z)=\frac{1+\sum_{k=1}^{m_{0}} \beta_{k}^{(n-1)} z^{k}}{1+\sum_{k=1}^{m_{0}} \beta_{k}^{(n)} z^{k}},
$$

where the constants $\beta_{k}^{(n)}$ are given by certain recursion formulas (see [1]).

Furthermore we shall need some well-known recursion formulas from the theory of continued fractions. Specializing to the present case and using the notation from $\S 1$, we have

$$
A_{n}(z) B_{n-1}(z)-A_{n-1}(z) B_{n}(z)=(-1)^{n-1} z^{n},
$$

$$
\begin{aligned}
A_{m}(z) / B_{m}(z) & \left(A_{n-1}(z)\left[1+d_{n} z+\frac{z}{1+d_{n+1} z}+\cdots+\frac{z}{1+d_{m} z}\right]+z A_{n-2}(z)\right) \\
& \cdot\left(B_{n-1}(z)\left[1+d_{n} z+\frac{z}{1+d_{n+1} z}+\cdots+\frac{z}{1+d_{m} z}\right]+z B_{n-2}(z)\right)^{-1}
\end{aligned}
$$

where $A_{k}(z)$ and $B_{k}(z)$ are polynomials, given by the recursion formulas

$$
\begin{aligned}
A_{-1}(z) & =1, \quad B_{-1}(z)=0, \\
A_{0}(z) & =1+d_{0} z, \quad B_{0}(z)=1, \\
A_{n}(z) & =\left(1+d_{n} z\right) A_{n-1}(z)+z A_{n-2}(z), \quad n=1,2,3, \cdots . \\
B_{n}(z) & =\left(1+d_{n} z\right) B_{n-1}(z)+z B_{n-2}(z),
\end{aligned}
$$

Immediately from (1), (2), and (8) it follows inductively

$$
\begin{aligned}
& A_{n-1}(z) f_{n}(z)+z A_{n-2}(z)=f_{0}(z) f_{1}(z) \cdots f_{n}(z), \\
& B_{n-1}(z) f_{n}(z)+z B_{n-2}(z)=f_{1}(z) f_{2}(z) \cdots f_{n}(z),
\end{aligned}
$$

and in particular,

$$
f_{0}(z)=\frac{A_{n-1}(z) f_{n}(z)+z A_{n-2}(z)}{B_{n-1}(z) f_{n}(z)+z B_{n-2}(z)} .
$$

Finally we rephrase Theorem 2.42 in [4] as

THEOREM 2. Let $\left\{a_{n}\right\}$ and $\left\{b_{n}\right\}$ be two sequences of complex-valued functions of the complex variable $z$, defined in some region $R$, and assume 
that they converge uniformly in $R$ to limit functions $a$ and $b$ respectively. Assume further the existence of a positive $\vartheta<1$ and two positive numbers $c$ and $C$, such that, in the whole region $R, c \leqq\left|\rho_{1}\right| \leqq C,\left|\rho_{2}\right| \rho_{1} \mid \leqq \vartheta$, where $\rho_{1}$ and $\rho_{2}$ denote the roots of the quadratic equation $\rho^{2}-b \rho-a=0$. Then there is an $N$, such that for $n \geqq N$ the continued fraction

$$
b_{n}(z)+\frac{a_{n+1}(z)}{b_{n+1}(z)}+\frac{a_{n+2}(z)}{b_{n+2}(z)}+\cdots
$$

converges uniformly in $R$ to a finite-valued limit function.

Proof of Theorem 1. (The first part of the proof is almost identical to the first part of Waadeland's proof of Lemma 1, while the second part strongly depends on the present conditions.)

Let

$$
1+d_{0} z+\frac{z}{1+d_{1} z}+\cdots+\frac{z}{1+d_{n} z}+\cdots
$$

be the $T$-fraction of $f_{0}$. By the hypothesis $\left\{d_{n}\right\}$ is convergent, and from Theorem 1 of [1] we know $d_{n} \rightarrow-1$ as $n \rightarrow \infty$. Therefore, putting $a_{n}(z)=z$, $b_{n}(z)=1+d_{n} z$, and $R=\{z ;|z|<\vartheta\}$ with $\vartheta \in(\theta, 1)$, we see that the convergence conditions of Theorem 2 are satisfied. Furthermore the inequalities are obviously valid since $\rho_{1}(z)=1$ and $\rho_{2}(z)=-z$ in the present case. Thus we conclude that there exists a number $N$ such that for $n \geqq N$ the $T$-fraction of $f_{n}$,

$$
1+d_{n} z+\frac{z}{1+d_{n+1} z}+\frac{z}{1+d_{n+2} z}+\cdots,
$$

converges uniformly on $D_{\theta}$ to a limit function $g$ (finite-valued). We assert that $g=f_{n}$ (restricted to $D_{\theta}$ ). Proof of this: The uniform convergence of the approximants of

$$
1+d_{n} z+\frac{z}{1+d_{n+1} z}+\frac{z}{1+d_{n+2} z}+\cdots
$$

implies, by local considerations, the continuity of $g$ (finite-valued). In particular $g$ is bounded on $D_{\theta}$, which in turn implies uniform boundedness of the sequence of approximants and thus regularity of

$$
1+d_{n} z+\frac{z}{1+d_{n+1} z}+\cdots+\frac{z}{1+d_{m} z}
$$

on $D_{\theta}$ for all $m \geqq M$ for some $M$. By Weierstrass we conclude that $g$ is holomorphic in $|z|<\theta$ and, for $k=1,2,3, \cdots$,

$$
g^{(k)}(0)=\lim _{m \rightarrow \infty} \frac{d^{k}}{d z^{k}}\left[1+d_{n} z+\frac{z}{1+d_{n+1} z}+\cdots+\frac{z}{1+d_{m} z}\right]_{z=0} .
$$


On the other hand, from the correspondence between $f_{n}(z)$ and (12) we have

$$
\begin{aligned}
& \lim _{m \rightarrow \infty} \frac{d^{k}}{d z^{k}}\left[1+d_{n} z+\frac{z}{1+d_{n+1} z}+\cdots+\frac{z}{1+d_{m} z}\right]=f_{n}^{(k)}(0), \\
& \quad k=1,2,3, \cdots, \\
& \text { (see Theorem } 2.1 \text { in [5]). }
\end{aligned}
$$

This agreement in Maclaurin series expansion of $f_{n}$ and $g$ shows that $f_{n}$ agrees with $g$ on $D_{\theta}$.

To finish the proof of the theorem, fix $n \geqq N$ and consider $m \geqq M$. We shall find it convenient to define functions $r_{m}$ on $D_{\theta}^{*}$ given by the formulas

$$
r_{m}(z)=1+d_{n} z+\frac{z}{1+d_{n+1} z}+\cdots+\frac{z}{1+d_{m} z}-f_{n}(z)
$$

Thus, from (7) and (11) the following holds in $D_{\theta}^{*}$ :

$$
\begin{aligned}
f_{0}(z) & -A_{m}(z) / B_{m}(z) \mid \\
= & \mid\left(z r_{m}(z)\left(A_{n-2}(z) B_{n-1}(z)-A_{n-1}(z) B_{n-2}(z)\right)\right) \\
& \cdot\left(\left(B_{n-1}(z) f_{n}(z)+z B_{n-2}(z)\right)\left(B_{n-1}(z)\left(f_{n}(z)+r_{m}(z)\right)+z B_{n-2}(z)\right)\right)^{-1} \mid .
\end{aligned}
$$

Applying (6), we finally get

$$
\begin{aligned}
\mid f_{0}(z) & -A_{m}(z) / B_{m}(z) \mid \\
= & \left(\left|r_{m}(z)\right|\left|z^{n}\right|\right) \\
& \cdot\left(\left|B_{n-1}(z) f_{n}(z)+z B_{n-2}(z)\right|\left|B_{n-1}(z)\left(f_{n}(z)+r_{m}(z)\right)+z B_{n-2}(z)\right|\right)^{-1} .
\end{aligned}
$$

We know that $r_{m}$ converges to 0 uniformly on $D_{\theta}$. Since $B_{n-1}, B_{n-2}$, and $f_{n}$ are holomorphic in $D_{\theta}$, we are done if we can show that $G$ defined by

$$
G(z)=B_{n-1}(z) f_{n}(z)+z B_{n-2}(z)
$$

has all its zeros among the poles of $f_{0}$. This, however, follows easily if we combine (5) and (10):

$$
B_{n-1}(z) f_{n}(z)+z B_{n-2}(z)=\frac{1+\sum_{k=1}^{m_{0}} \beta_{k}^{(0)} z^{k}}{1+\sum_{k=1}^{m_{0}} \beta_{k}^{(n)} z^{k}}
$$

\section{REFERENCES}

1. K. Hag, A theorem on T-fractions corresponding to a rational function, Proc. Amer. Math. Soc. 25 (1970), 247-253. MR 41 \#3723.

2. W. B. Jones, Contributions to the theory of Thron continued fractions, Ph.D. Thesis, Vanderbilt University, Nashville, Tennessee, 1963, 68 pp.

3. W. B. Jones and W. J. Thron, Further properties of T-fractions, Math. Ann. 166 (1966), 106-118. MR 34 \#319. 
4. O. Perron, Die Lehre von den Kettenbrüchen, Verbesserte und erweiterte Aufl., Band II, Analytisch-funktionentheoretische Kettenbrüche, Teubner Verlagsgesellschaft, Stuttgart, 1957. MR 19, 25.

5. W. J. Thron, Some properties of continued fractions $1+d_{0} z+K\left(z /\left(1+d_{n} z\right)\right)$, Bull. Amer. Math. Soc. 54 (1948), 206-218. MR 9, 508.

6. H. Waadeland, On T-fractions of functions holomorphic and bounded in a circular disc, Norske Vid. Selsk. Skr. (Trondheim) 1964, no. 8, 19 pp. MR 31 \#1364.

Department of Mathematics, University of Trondheim, Trondheim, Norway

Department of Mathematics, University of Michigan, Ann Arbor, Michigan 48104 